\title{
Challenges in endocrinology: moving from the post-genomic era, into the nano-world and beyond
}

\author{
Jeff M. P. Holly* \\ School of Clinical Science, Southmead Hospital, University of Bristol, Bristol, UK \\ *Correspondence: jeff.holly@bristol.ac.uk
}

Major new health issues confront us; some of which arise from our modern lifestyle and its consequent energy imbalance. In addition to obesity and diabetes, metabolic disturbances will soon overtake smoking as the main avoidable cause of cancer. Reductions in infectious diseases and infant mortality together with people living longer result in population growth and an increased need for effective population control: a clear challenge for reproductive endocrinology. Many more people living to old age increases the prevalence of all conditions associated with aging. Until recently aging was generally considered to be due to wear-and-tear and cumulative tissue damage. Studies from experimental genetics have however now clearly demonstrated that aging is regulated by hormonal pathways and that longevity can be dramatically extended; raising the potential for endocrine-based interventions for many age-related conditions. Assessing and treating endocrine disorders in the very elderly or considering endocrine interventions to counter frailty is however challenging because of limited normative data and a lack of understanding of normal endocrinology in this age group. A further challenge will be to balance quantity-of-life with quality-of-life.

Rodent models have been widely used in endocrinology but there are concerns regarding their use as models of these new human conditions that are a consequence of metabolic disorders related to geneenvironment interactions. Laboratories use genetically homogeneous rodent colonies and hence examine the effect of exposures on a single genotype. Human populations however comprise infinitely varied genotypes which can respond in many different ways to the same exposures; even in the most obesogenic environment only some individuals become obese. In addition the standard rodent experiment compares an intervention group to an untreated control group. These control animals however live a sedentary life with continuous access to food, within confined containers in standardized conditions with constant day-night cycles and hence no environmental stimuli. As a result these "control" animals are relatively overweight, insulin resistant and live much shorter lives than "normal" rodents living in their natural habitats that exercise considerably more and feed intermittently (Martin et al., 2010). Interventions that are perceived to induce metabolic advantage in such experiments are in many cases just correcting these abnormalities present in the control animals. This experimental paradigm may have had many unwitting consequences; for example many new cancer therapies exhibit efficacy in animal studies but then prove to be ineffective in human clinical trials. Is this because tumor development is different in the metabolically moribund animals used in the preclinical trials? Similarly most cell culture experiments are traditionally performed in very hyperglycemic media which can alter many cell responses. A challenge will be to develop more appropriate experimental models.

Historically endocrinology studied a few discrete endocrine glands but it is now clear that most tissues release soluble signals. Adipose tissue, once regarded as just a passive energy store, is now recognized as a dynamic source of hormones such as leptin and adiponectin. The gastrointestinal tract secretes ghrelin and incretins and muscle is a source of myokines. With ever growing complexities each of the endocrine systems has become a field within itself, being an expert in thyroid endocrinology for example, involves integrating considerable information from structural chemistry through to clinical management. Integrating all of this information for more than one system is challenging. In the body, cells never see any hormone in isolation; they are exposed to many varying signals. Experiments usually test how one variable affects one response, examining a dynamic response adds a third-dimension: conceptualizing in more than $3 \mathrm{D}$ has constrained more complex experiments. To move the experimental paradigm closer to reality will require the use of techniques such as mathematical modeling and network analysis.

Technical advances are now occurring so rapidly that interpretation struggles to keep apace. It is now possible to examine virtually all of the genetic variants across the entire genome and their association with endocrine disorders such as obesity and diabetes. Early genome-wide association studies indicate that large numbers of genes generally each contribute small effects to these common conditions and most are dependent upon gene-environment interactions. The first robust association with obesity was the fat mass and obesityassociated gene, FTO (Frayling et al., 2007). More recently it was reported that this association was not present in an older population not exposed to the current obesogenic environment (Jacobsson et al., 2009) and the association could also be overcome in young people who meet recommended levels of daily physical activity (Ruiz et al., 2010); implying that FTO was not causal but was a susceptibility gene affecting the response to energy imbalance. Changes in all of the genes that respond to a hormone can now be arrayed and this can even be examined dynamically using gene expression dynamic imaging. Further complexity has emerged from epigenetics: many genes can be reprogrammed by a variety of mechanisms enabling adaptation to altered circumstances such as changing availability of food and water. As these functions are hormonally controlled it is not surprising that these controls can be epigenetically reprogrammed. Such programming continues from in utero at least throughout childhood. Pediatric endocrinologists need to understand these adaptations and their long-term consequences. Childhood obesity indicates disturbed metabolism at an age when there is still plasticity and the potential for reprogramming endocrine systems. This may lead to long-term consequences 
in addition to all of the recognized sequalae of obesity that normally develops in midlife. In addition to genetics and epigenetics, there have been important developments in RNA-biology with the discovery of microRNA and multiple RNA binding proteins and splicing factors. Advances in proteomics and metabolomics are also yielding unprecedented volumes of information regarding hormone processing and hormonal effects.

Advances in chemistry and physics have opened exciting new windows to the worlds of cellular and molecular endocrinology. We can now actually see the subtle twists and turns that occur when a hormone engages with its receptor and study these events with techniques such as single-molecule fluorescence microscopy, FRET, FRAP, and FLIM (fluorescence energy transfer, fluorescence recovery after photobleaching, and fluorescence lifetime imaging microscopy). Quantum dots (Qdots) are extremely bright and photostable nano-sized crystalline clusters making them useful probes for fluorescence imaging, facilitating multiplexing, and with particular advantages for FRET and two-photon excitation microscopy (Pinaud et al., 2010). Atomic Force Microscopy can be used in combination with techniques such as scanning fluorescence correlation spectroscopy and fluorescence lifetime correlation spectroscopy to study ligand/ receptor interactions on live cells (Chen and Irudayaraj, 2010). Labeled nanoparticles can be manipulated with optical tweezers for 3D nanoscale imaging which with video-goggles enable the viewer to move around cells or molecular structures in the virtual nanoworld. Touch-pads can be used to manipulate ligands onto receptors and then gently tug to assess binding forces. When linked to tactile-gloves this even enables the investigator to feel the surface of cells or large molecular structures. A challenge will be to translate this new molecular insight into information that may benefit patients, such as better design of molecular mimetics and antagonists.

There have been similar advances in clinical imaging techniques including ultrasound, scintigraphy, angiography, computed tomography (CT) including single-photon emission CT (SPECT), MRI, and PET; aiding patient evaluation, diagnosis, and monitoring response. Functional imaging is evolving with new probes: for example imaging for hyperparathyroidism is now possible with either dual-isotope subtraction imaging or single-isotope dual-phase imaging and the distinction of parathyroid and thyroid glands provided by SPECT can be combined with anatomical information provided by CT (Eslamy and Ziessman, 2008). The application of nanoparticles and Qdots will confer immunohistochemical specificity to clinical imaging; already used for experimental animals and will be translated to the clinic as soon as toxicology issues have been solved (Bentolila et al., 2009). This will enable hormones, drugs, antibodies, and cells to be tagged with nanoparticles and tracked within the body; such as herceptin-targeted MRI imaging of breast cancer cells (Huh et al., 2005) and VEGF-conjugated Qdots for PET imaging of angiogenesis (Chen et al., 2008). Multifunctional nanoparticles will enable multifunctional imaging and other developments such as metallic nanoparticles with photothermal properties for combined targeted imaging and laser ablation therapy. Nanostructures mimicking biological systems are being developed including motorized nanoparticles and more complex structures with DNA (Gu et al., 2010) or RNA (Guo, 2005) based assembly. Such complex nanostructures could eventually carry antibodies linked to Qdots or paramagnetic particles for target imaging and subunits that induce receptor-mediated endocytosis, disruption of endosomes and release of drugs, and a final subunit with a further label for imaging of the planned outcome such as apoptosis.

Endocrinology is now central to the major current health issues of aging, population control, and the pandemics of obesity, diabetes, and cancer. I have described some examples of exciting new technologies: applying these and making sense of the mountains of new data that they will generate and translating all of this into new understanding and benefits for patients is the challenge for endocrinology.

\section{REFERENCES}

Bentolila, L. A., Ebenstein, Y., and Weiss, S. (2009). Quantum dots for in vivo small-animal imaging. J. Nucl. Med. 50, 493-496.

Chen, J., and Irudayaraj, J. (2010). Fluorescence lifetime cross correlation spectroscopy resolves EGFR and antagonist interaction in live cells. Anal. Chem. 82, 6415-6421.

Chen, K., Li, Z. B., Wang, H., Cai, W., and Chen, X. (2008).Dual-modality optical and positron emission tomography imaging of vascular endothelial growth factor receptor on tumor vasculature using quantum dots. Eur. J. Nucl. Med. Mol. Imaging 35, 2235-2244.

Eslamy, H. K., and Ziessman, H. A. (2008). Parathyroid scintigraphy in patients with primary hyperparathyroidism: 99mTc sestamibi SPECT and SPECT/CT. Radiographics 28, 1461-1476.

Frayling, T.M., Timpson, N. J., Weedon, M. N.,Zeggini, E., Freathy, R. M., Lindgren, C. M., Perry, J. R., Elliott, K. S., Lango, H., Rayner, N. W., Shields, B., Harries, L. W., Barrett, J.C., Ellard, S., Groves, C. J., Knight, B., Patch,A. M., Ness, A. R., Ebrahim, S., Lawlor, D. A., Ring, S. M., Ben-Shlomo, Y., Jarvelin, M. R., Sovio, U., Bennett, A. J., Melzer, D., Ferrucci, L., Loos, R. J., Barroso, I., Wareham, N. J., Karpe, F., Owen, K. R., Cardon, L. R., Walker, M., Hitman, G. A., Palmer, C. N., Doney, A. S., Morris, A. D., Smith, G. D., Hattersley, A. T., and McCarthy, M. I. (2007). A common variant in the FTO gene is associated with body mass index and predisposes to childhood and adult obesity. Science 316, 889-894.

Gu, H., Chao, J., Xiao, S. J., and Seeman, N. C. (2010). A proximity-based programmable DNA nanoscale assembly line. Nature 465, 202-205.

Guo, P. (2005). RNA nanotechnology: engineering, assembly and applications in detection, gene delivery and therapy. J. Nanosci. Nanotechnol. 5, 1964-1982.

Huh, Y.M., Jun, Y. W., Song, H. T., Kim, S., Choi, J. S., Lee, J. H., Yoon, S., Kim, K. S., Shin, J. S., Suh, J. S., and Cheon, J. (2005). In vivo magnetic resonance detection of cancer by using multifunctional magnetic nanocrystals. J. Am. Chem. Soc. 127, 12387-12391.

Jacobsson, J. A., Risérus, U., Axelsson, T., Lannfelt, L., Schiöth, H. B., and Fredriksson, R. (2009). The common FTO variant rs9939609 is not associated with BMI in a longitudinal study on a cohort of Swedish men born 1920-1924. BMC Med. Genet. 10, 131. doi: 10.1186/1471-2350-10-131

Martin, B., Ji., S., Maudsley, S., and Mattson, M. P. (2010). "Control" laboratory rodents are metabolically morbid: why it matters. Proc. Natl. Acad. Sci. U.S.A. 107, 6127-6133.

Pinaud, F., Clarke, S., Sittner, A., and Dahan, M. (2010). Probing cellular events, one quantum dot at a time. Nat. Methods 7, 275-285.

Ruiz, J. R., Labayen, I., Ortega, F. B., Legry, V., Moreno, L.A., Dallongeville, J., Martínez-Gómez,D., Bokor,S., Manios, Y., Ciarapica, D., Gottrand, F., De Henauw, S., Molnár, D., Sjöström, M., Meirhaeghe, A., and HELENA Study Group. (2010). Attenuation of the effect of the FTO rs9939609 polymorphism on total and central body fat by physical activity in adolescents: the HELENA study. Arch. Pediatr. Adolesc. Med. 164, 328-333.

Received: 05 November 2010; accepted: 22 December 2010; published online: 08 February 2011.

Citation: Holly JMP (2011) Challenges in endocrinology: moving from the post-genomic era, into the nanoworld and beyond. Front. Endocrin. 1:11. doi: 10.3389/ fendo.2010.00011

Copyright $\odot 2011$ Holly. This is an open-access article subject to an exclusive license agreement between the authors and Frontiers Media SA, which permits unrestricted use, distribution, and reproduction in any medium, provided the original authors and source are credited. 